\title{
The Cauchy Problem for a Fifth-Order Dispersive Equation
}

\author{
Hongjun Wang, Yongqi Liu, and Yongqiang Chen \\ College of Mathematics and Information Science, Henan Normal University, Xinxiang, Henan 453007, China
}

Correspondence should be addressed to Hongjun Wang; hsdwhj@163.com

Received 21 January 2014; Accepted 8 February 2014; Published 27 March 2014

Academic Editor: Changsen Yang

Copyright (C) 2014 Hongjun Wang et al. This is an open access article distributed under the Creative Commons Attribution License, which permits unrestricted use, distribution, and reproduction in any medium, provided the original work is properly cited.

This paper is devoted to studying the Cauchy problem for a fifth-order equation. We prove that it is locally well-posed for the initial data in the Sobolev space $H^{s}(\mathbf{R})$ with $s \geq 1 / 4$. We also establish the ill-posedness for the initial data in $H^{s}(\mathbf{R})$ with $s<1 / 4$. Thus, the regularity requirement for the fifth-order dispersive equations $s \geq 1 / 4$ is sharp.

\section{Introduction}

In this paper, we consider the Cauchy problem and existence of solitary waves of the following fifth-order dispersive equation:

$$
\begin{gathered}
\partial_{t} u-\partial_{t} \partial_{x}^{2} u+\partial_{x}^{3} u-\partial_{x}^{5} u+\frac{1}{3} \partial_{x}\left(u^{3}\right)-\frac{1}{3} \partial_{x}^{3}\left(u^{3}\right) \\
+\partial_{x}\left[\frac{5}{3} u^{3}+u\left(\partial_{x} u\right)^{2}\right]=0 \\
u(x, 0)=\phi .
\end{gathered}
$$

Obviously, (1) can be seen as the higher modification of the following dispersive equation:

$$
\begin{gathered}
\partial_{t} u-\partial_{t} \partial_{x}^{2} u+\frac{1}{3} \partial_{x}\left(u^{3}\right)-\frac{1}{3} \partial_{x}^{3}\left(u^{3}\right) \\
+\partial_{x}\left[\frac{5}{3} u^{3}+u\left(\partial_{x} u\right)^{2}\right]=0 .
\end{gathered}
$$

We recall that

$$
u_{t}+(a(u))_{x}-u_{x t t}-\left(b^{\prime}(u) \frac{u_{x}^{2}}{2}+b(u) u_{x x}\right)_{x}=0
$$

where

$$
b(u)=u^{p}, \quad a(u)=2 k u+\frac{p+2}{2} u^{p+1},
$$

which has been studied in [1], is the generalized CamassaHolm equation. In fact, Hakkaev and Kirchev [1] studied the local well-posedness and orbital stability and instability of (4) with the aid of the pseudoparabolic regularization and spectral analysis. Obviously, when $p=2$, (4) is the wellknown Camassa-Holm equation; when $p=3$ and $k=0$, (4) becomes (3). Some people consider the Cauchy problem for the higher modification of the nonlocal form of CamassaHolm equation; we refer the readers to [2-5].

By acting $\left(1-\partial_{x}^{2}\right)^{-1}$ on both sides of (1), we obtain the following equivalent form:

$$
\partial_{t} u+\partial_{x}^{3} u+\frac{1}{3} \partial_{x}\left(u^{3}\right)+\left(1-\partial_{x}^{2}\right)^{-1} \partial_{x}\left[\frac{5}{3} u^{3}+u\left(\partial_{x} u\right)^{2}\right]=0 .
$$

Obviously, (6) is a modification of $\mathrm{mKdV}$ equation

$$
\partial_{t} u+\partial_{x}^{3} u+\frac{1}{3} \partial_{x}\left(u^{3}\right)=0
$$

which has been intensively studied; we refer the readers to [611]. In [8], the authors proved that (7) is locally well-posed for the initial data in $H^{s}(\mathbf{R})$ with $s \geq 1 / 4$. The regularity requirement for the modified $\mathrm{KdV}$ equation $s \geq 1 / 4$ is sharp; see [9]. In [11], by using the $I$-method which can be seen in [11-14] and Mirua transformation which can be seen in [15], the authors proved that the modified $\mathrm{KdV}$ equation is globally well-posed for the initial data in $H^{s}(\mathbf{R})$ with $s>1 / 4$. In [16], by using the dyadic bilinear estimates and resolution spaces which can be seen in $[17,18]$, the author proved that the modified KdV equation is globally well-posed for the initial data in $H^{s}(\mathbf{R})$ with $s=1 / 4$. 
In this paper, by using the Fourier restriction norm method introduced in [19], we prove that (1) and (2) are locally well-posed for the initial data in $H^{s}(\mathbf{R})$ with $s \geq 1 / 4$. When $\phi \in H^{s}(\mathbf{R}), s \geq 1$, we prove that the Cauchy problem for (1) is globally well-posed in $H^{s}(\mathbf{R})$. By using the general well-posedness principle proposed by [20], we establish the ill-posedness for the initial data in $H^{s}(\mathbf{R})$ with $s<1 / 4$. Thus, the requirement for regularity $s \geq 1 / 4$ is sharp.

Before stating the main results, we introduce some notations and definitions. We use $X \sim Y$ when $C_{1}|X| \leq|Y| \leq$ $C_{2}|X|$, where $C_{j}>0(j=1,2)$; we use $X \gg Y$ when $|X|>C|Y|$, where $C$ is some positive number which is larger than $2 .\langle\xi\rangle^{s}=\left(1+\xi^{2}\right)^{s / 2}$ for any $\xi \in \mathbf{R}$, and $\mathscr{F} u$ is the Fourier transform of $u$ with respect to its all variables. $\mathscr{F}_{x} u$ is the Fourier transform of $u$ with respect to its space variables. Denote $D_{x}=\left(-\partial_{x}^{2}\right)^{1 / 2} \cdot \mathcal{S}\left(\mathbf{R}^{n}\right)(n=1,2,3)$ is the Schwartz space and $\mathcal{S}^{\prime}\left(\mathbf{R}^{n}\right)$ is its dual space. $H^{s}(\mathbf{R})$ is the usual Sobolev space with norm $\|f\|_{H^{s}(\mathbf{R})} \triangleq\left\|\langle\xi\rangle^{s} \mathscr{F}_{x} f\right\|_{L_{\xi}^{2}(\mathbf{R})}$. For any $s, \alpha \in \mathbf{R}, X_{s, \alpha}\left(\mathbf{R}^{2}\right)$ is the Bourgain space with phase function $\phi(\xi)=-\xi^{3}$. That is, a function $u(x, t)$ in $\delta^{\prime}\left(\mathbf{R}^{2}\right)$ belongs to $X_{s, \alpha}\left(\mathbf{R}^{2}\right)$ if and only if

$$
\|u\|_{X_{s, \alpha}\left(\mathbf{R}^{2}\right)} \triangleq\left\|\langle\xi\rangle^{s}\left\langle\tau-\xi^{3}\right\rangle^{\alpha} \mathscr{F} u(\xi, \tau)\right\|_{L_{\tau \xi}^{2}\left(\mathbf{R}^{2}\right)}<\infty .
$$

For any given interval $L, X_{s, \alpha}(\mathbf{R} \times L)$ is the space of the restriction of all functions in $X_{s, \alpha}\left(\mathbf{R}^{2}\right)$ on $\mathbf{R} \times L$, and for $u \in X_{s, \alpha}(\mathbf{R} \times L)$ its norm is

$$
\|u\|_{X_{s, \alpha}(\mathbf{R} \times L)}=\inf \left\{\|U\|_{X_{s, \alpha}\left(\mathbf{R}^{2}\right)} ;\left.U\right|_{\mathbf{R} \times L}=u\right\} .
$$

We write $X_{s, \alpha}^{T}$ for $X_{s, \alpha}(\mathbf{R} \times L)$ when $L=[0, T]$. Let $\sigma=\tau-$ $\xi^{3}, \sigma_{j}=\tau_{j}-\xi_{j}^{3}(j=1,2,3)$.

The main results of this paper are as follows.

Theorem 1. The Cauchy problem for (1) is locally well-posed for the initial data $\phi$ in $H^{s}(\mathbf{R})$ with $s \geq 1 / 4$.

Theorem 2. Let $s<1 / 4$. Then (1) and (2) are ill-posed in $H^{s}(\mathbf{R})$ in the sense that the solution map $S_{t}$ of the Cauchy problem for (1) is not Lipschitz continuous at zero. More precisely, for any $T>0$, the solution map

$$
S_{t}: \phi \in H^{s}(\mathbf{R}) \longrightarrow u \in C\left([0, T] ; H^{s}(\mathbf{R})\right)
$$

is not Lipschitz continuous at zero.

The rest of this paper is arranged as follows. In Section 2, we make some preliminaries. In Section 3, we establish two crucial trilinear estimates. In Section 4, we prove Theorem 1. In Section 5, we prove Theorem 2.

\section{Preliminaries}

Lemma 3. Let $b>1 / 2$. Then

$$
\begin{gathered}
\|u\|_{L_{x t}^{4}} \leq C\|u\|_{X_{0,2 b / 3}}, \\
\left\|D_{x}^{-1 / 4} u\right\|_{L_{x}^{4} L_{t}^{\infty}} \leq C\|u\|_{X_{0, b}},
\end{gathered}
$$

$$
\begin{gathered}
\left\|D_{x} u\right\|_{L_{x}^{\infty} L_{t}^{2}} \leq C\|u\|_{X_{0, b}}, \\
\|u\|_{L_{x t}^{6}} \leq C\|u\|_{X_{0,8 b / 9}} .
\end{gathered}
$$

Proof. From [21], we have

$$
\|u\|_{L_{x t}^{8}} \leq C\|u\|_{X_{0, b}} .
$$

From Plancherel's identity, we have

$$
\|u\|_{L_{x t}^{2}}=C\|u\|_{X_{0, b}} .
$$

Interpolating (15) with (16) yields (14). Equations (11)-(13) can be seen in $[21,22]$.

Lemma 4. For $0<\delta<1, s \in \mathbf{R}$, and $1 / 2<b \leq 1$, we have

$$
\left\|\psi_{\delta}(t) W(t) u_{0}\right\|_{X_{s, b}} \leq C \delta^{1 / 2-b}\left\|u_{0}\right\|_{H^{s}}
$$

and for $-1 / 2<b^{\prime} \leq 0 \leq b \leq b^{\prime}+1$, we have

$$
\left\|\psi_{\delta}(t) \int_{0}^{t} W(t-\tau) f(\tau) d \tau\right\|_{X_{s, b}} \leq C \delta^{1+b^{\prime}-b}\|f\|_{X_{s, b^{\prime}}} .
$$

Equations (17) and (18) can be seen in $[6,23]$.

Lemma 5. Let $\xi=\xi_{1}+\xi_{2}+\xi_{3}$. Then

$$
\xi^{3}-\xi_{1}^{3}-\xi_{2}^{3}-\xi_{3}^{3}=3\left(\xi_{1}+\xi_{2}\right)\left(\xi_{1}+\xi_{3}\right)\left(\xi_{2}+\xi_{3}\right) .
$$

Lemma 5 can be found in [11].

Lemma 6. Let $I^{s}$ denote the Riesz potential of order $-s$ and $b>1 / 2 \geq s \geq 0, \tilde{b}>1 / 6+2 s / 3$. Then the following estimate holds true:

$$
\left\|I^{s} I_{-}^{s}(u, v)\right\|_{L_{x t}^{2}} \leq C\|u\|_{X_{0, b}}\|v\|_{X_{0, \tilde{b}}},
$$

where

$$
\mathscr{F}_{x} I_{-}^{s}(u, v)(\xi)=\int_{\xi=\xi_{1}+\xi_{2}}\left|\xi_{1}-\xi_{2}\right|^{s} \mathscr{F}_{x} u\left(\xi_{1}\right) \mathscr{F}_{x} v\left(\xi_{2}\right) d \xi_{1} .
$$

In particular, when $s=1 / 2$ and $b>1 / 2$, we have

$$
\left\|I^{1 / 2} I_{-}^{1 / 2}(u, v)\right\|_{L_{x t}^{2}} \leq C\|u\|_{X_{0,(1 / 2)+}}\|v\|_{X_{0,(1 / 2)+}} .
$$

Lemma 6 can be seen in [6].

\section{Trilinear Estimates}

In this section, we will prove two crucial trilinear estimates.

Lemma 7. Let $s \geq 1 / 4, b=1 / 2+2 \epsilon, b^{\prime}=-1 / 2+3 \epsilon, 0<\epsilon \ll 1$. Then

$$
\left\|\partial_{x}\left(1-\partial_{x}^{2}\right)^{-1}\left[u_{1}\left(\partial_{x} u_{2}\right)\left(\partial_{x} u_{3}\right)\right]\right\|_{X_{s, b^{\prime}}} \leq C \prod_{j=1}^{3}\left\|u_{j}\right\|_{X_{s, b}} .
$$


Proof. Let

$$
\begin{aligned}
F_{j}\left(\xi_{j}, \tau_{j}\right) & =\left\langle\xi_{j}\right\rangle^{s}\left\langle\sigma_{j}\right\rangle^{b} \mathscr{F} u_{j}\left(\xi_{j}, \tau_{j}\right) \quad(j=1,2,3), \\
F(\xi, \tau) & =\langle\xi\rangle^{-s}\langle\sigma\rangle^{-b^{\prime}} \mathscr{F} u(\xi, \tau) .
\end{aligned}
$$

By duality and Plancherel's identity, to derive (23), it suffices to prove

$$
\begin{aligned}
& \int_{\mathbf{R}^{2}} \int_{\substack{\xi=\xi_{1}+\xi_{2}+\xi_{3} \\
\tau=\tau_{1}+\tau_{2}+\tau_{3}}}|F| \prod_{j=1}^{3}\left|F_{j}\right| \\
& \quad \times K\left(\xi_{1}, \tau_{1}, \xi_{2}, \tau_{2}, \xi_{3}, \tau_{3}, \xi, \tau\right) d \xi_{1} d \tau_{1} d \xi_{2} d \tau_{2} d \xi d \tau \\
& \leq C\|F\|_{L_{\xi \tau}^{2}} \prod_{j=1}^{3}\left\|F_{j}\right\|_{L_{\xi_{\tau}}^{2}}
\end{aligned}
$$

where

$$
\begin{aligned}
& K\left(\xi_{1}, \tau_{1}, \xi_{2}, \tau_{2}, \xi, \tau\right) \\
& =\frac{|\xi|\left(\left|\xi_{2}\right|\left|\xi_{3}\right|+\left|\xi_{1}\right|\left|\xi_{2}\right|+\left|\xi_{1}\right|\left|\xi_{3}\right|\right)\langle\xi\rangle^{s}}{\left(1+\xi^{2}\right)\langle\sigma\rangle^{-b^{\prime}} \prod_{j=1}^{3}\left\langle\xi_{j}\right\rangle^{s}\langle\sigma\rangle^{b}} .
\end{aligned}
$$

Without loss of generality, we may assume that $F_{j}\left(\xi_{j}, \tau_{j}\right)(j=$ $1,2,3) \geq 0$ and $F(\xi, \tau) \geq 0$. By the symmetry among $\left|\xi_{1}\right|$, $\left|\xi_{2}\right|,\left|\xi_{3}\right|$, without loss of generality, we may assume that $\left|\xi_{1}\right| \geq$ $\left|\xi_{2}\right| \geq\left|\xi_{3}\right|$. Obviously, $\left\{\left|\xi_{1}\right| \geq\left|\xi_{2}\right| \geq\left|\xi_{3}\right|\right\} \subseteq \bigcup_{j=1}^{6} \Omega_{j}$, where

$$
\begin{aligned}
& \Omega_{1}=\left\{8 \geq\left|\xi_{1}\right| \geq\left|\xi_{2}\right| \geq\left|\xi_{3}\right|\right\}, \\
& \Omega_{2}=\left\{\left|\xi_{1}\right| \geq 8,\left|\xi_{1}\right| \gg\left|\xi_{2}\right| \geq\left|\xi_{3}\right|\right\}, \\
& \Omega_{3}=\left\{\left|\xi_{1}\right| \geq 8,\left|\xi_{1}\right| \sim\left|\xi_{2}\right| \gg\left|\xi_{3}\right|,\right. \\
& \left.\left|\xi_{1}\right| \geq\left|\xi_{2}\right| \geq\left|\xi_{3}\right|,\left|\xi_{3}\right| \leq 8\right\}, \\
& \Omega_{4}=\left\{\left|\xi_{1}\right| \geq 8,\left|\xi_{1}\right| \sim\left|\xi_{2}\right| \gg\left|\xi_{3}\right|\right. \text {, } \\
& \left.\left|\xi_{1}\right| \geq\left|\xi_{2}\right| \geq\left|\xi_{3}\right| \geq 8\right\} \text {, } \\
& \Omega_{5}=\left\{\left|\xi_{1}\right| \geq 8,\left|\xi_{1}\right| \sim\left|\xi_{2}\right| \sim\left|\xi_{3}\right|,\right. \\
& \left.\left|\xi_{1}\right| \geq\left|\xi_{2}\right| \geq\left|\xi_{3}\right|,|\xi| \geq \frac{\left|\xi_{3}\right|}{3}\right\}, \\
& \Omega_{6}=\left\{\left|\xi_{1}\right| \geq 8,\left|\xi_{1}\right| \sim\left|\xi_{2}\right| \sim\left|\xi_{3}\right|,\right. \\
& \left.\left|\xi_{1}\right| \geq\left|\xi_{2}\right| \geq\left|\xi_{3}\right|,|\xi| \leq \frac{\left|\xi_{3}\right|}{3}\right\} .
\end{aligned}
$$

In this lemma, integrals over the subregion $\Omega_{k}$ 's are, respectively, denoted as $J_{k}(1 \leq k \leq 6, k \in \mathbf{Z})$. Consider

$$
\mathscr{F} f_{j}=\frac{F_{j}}{\left\langle\sigma_{j}\right\rangle^{b}} \quad(j=1,2,3), \quad \mathscr{F} f=\frac{F}{\langle\sigma\rangle^{-b^{\prime}}} .
$$

(1) Subregion $\left\{8 \geq\left|\xi_{1}\right| \geq\left|\xi_{2}\right| \geq\left|\xi_{3}\right|\right\}$. In this subregion, since $s \geq 1 / 4$ and $|\xi| \leq 3$, we have

$$
K_{1}\left(\xi_{1}, \tau_{1}, \xi_{2}, \tau_{2}, \xi, \tau\right) \leq \frac{C}{\langle\sigma\rangle^{-b^{\prime}} \prod_{j=1}^{3}\left\langle\sigma_{j}\right\rangle^{b}}
$$

By using Cauchy Schwartz's inequality and Plancherel's identity as well as Hölder's inequality, (11), $2 b / 3<b$ and $2 b / 3<$ $-b^{\prime}$, we derive

$$
\begin{aligned}
J_{1} & \leq C \int_{\mathbf{R}^{2}} \int_{\substack{\xi=\xi_{1}+\xi_{2}+\xi_{3}+\tau_{3} \\
\tau=\tau^{-b^{\prime}} \prod_{j=1}^{3}\left\langle\sigma_{j}\right\rangle^{b}}} \frac{F \xi_{1}^{3} d \tau_{1} d \xi_{2} d \tau_{2} d \xi d \tau}{\langle\sigma\rangle_{j}} \\
& \leq C\|f\|_{L_{x t}^{4}} \prod_{j=1}^{3}\left\|f_{j}\right\|_{L_{x t}^{4}}
\end{aligned}
$$

$$
\begin{aligned}
& \leq C\|f\|_{X_{0,2 b / 3}} \prod_{j=1}^{3}\left\|f_{j}\right\|_{X_{0,2 b / 3}} \\
& \leq C\|F\|_{L_{\xi \tau}^{2}} \prod_{j=1}^{3}\left\|F_{j}\right\|_{L_{\xi \tau}^{2}} .
\end{aligned}
$$

(2) Subregion $\left\{\left|\xi_{1}\right| \geq 8,\left|\xi_{1}\right| \gg\left|\xi_{2}\right| \geq\left|\xi_{3}\right|\right\}$. In this subregion, $|\xi| \sim\left|\xi_{1}\right|$ and $\left|\xi_{1}\right| \gg\left|\xi_{2}\right|$, since $s \geq 1 / 4$, we have

$$
\begin{aligned}
K_{1}\left(\xi_{1}, \tau_{1}, \xi_{2}, \tau_{2}, \xi, \tau\right) & \leq C \frac{\left|\xi_{2}\right|}{\langle\sigma\rangle^{-b^{\prime}} \prod_{j=1}^{3}\left\langle\sigma_{j}\right\rangle^{b}} \\
& \leq C \frac{\left|\xi_{1}^{2}-\xi_{2}^{2}\right|^{1 / 2}}{\langle\sigma\rangle^{-b^{\prime}} \prod_{j=1}^{3}\left\langle\sigma_{j}\right\rangle^{b}}
\end{aligned}
$$

By using Cauchy-Schwartz's inequality and Plancherel's identity, (22) of Lemma 6 , (11), $2 b / 3<b$, and $2 b / 3<-b^{\prime}$, since $s \geq 1 / 4$, we obtain

$$
\begin{aligned}
& J_{2} \leq C \int_{\mathbf{R}^{2}} \int_{\substack{\xi=\xi_{1}+\xi_{2}+\xi_{3} \\
\tau=\tau_{1}+\tau_{2}+\tau_{3}}} \frac{\left|\xi_{1}^{2}-\xi_{2}^{2}\right|^{1 / 2} F \prod_{j=1}^{3} F_{j}}{\langle\sigma\rangle^{-b^{\prime}} \prod_{j=1}^{3}\left\langle\sigma_{j}\right\rangle^{b}} d \xi_{1} d \tau_{1} d \xi_{2} d \tau_{2} d \xi d \tau \\
& \leq C\left\|I\left(f_{1,2}\right)\right\|_{L_{x t}^{2}}\|f\|_{L_{x t}^{4}}\left\|f_{3}\right\|_{L_{x t}^{4}} \\
& \leq C\|f\|_{X_{0,2 b / 3}}\left\|f_{3}\right\|_{X_{0,2 b / 3}} \prod_{j=1}^{2}\left\|f_{j}\right\|_{X_{0, b}} \\
& \leq C\|F\|_{L_{\xi \tau}^{2}} \prod_{j=1}^{3}\left\|F_{j}\right\|_{L_{\xi \tau}^{2}}
\end{aligned}
$$


(3) Subregion $\left\{\left|\xi_{1}\right| \geq 8,\left|\xi_{1}\right| \sim\left|\xi_{2}\right| \gg\left|\xi_{3}\right|,\left|\xi_{3}\right| \leq 8\right\}$. In this subregion, since $s \geq 1 / 4$ and $|\xi| \leq C\left|\xi_{2}\right|$, we have

$$
\begin{aligned}
K_{1}\left(\xi_{1}, \tau_{1}, \xi_{2}, \tau_{2}, \xi, \tau\right) & \leq C \frac{\left|\xi_{2}\right|^{1-s}}{\langle\sigma\rangle^{-b^{\prime}} \prod_{j=1}^{3}\left\langle\sigma_{j}\right\rangle^{b}} \\
& \leq C \frac{\left|\xi_{2}^{2}-\xi_{3}^{2}\right|^{1 / 2}}{\langle\sigma\rangle^{-b^{\prime}} \prod_{j=1}^{3}\left\langle\sigma_{j}\right\rangle^{b}} .
\end{aligned}
$$

This case can be treated similarly to $J_{2}$.

(4) Subregion $\left\{\left|\xi_{1}\right| \geq 8,\left|\xi_{1}\right| \sim\left|\xi_{2}\right| \gg\left|\xi_{3}\right| \geq 8\right\}$. In this subregion $\left|\xi_{1}\right| \sim\left|\xi_{2}\right|$ and $|\xi| \leq C\left|\xi_{1}\right|$.

When $s \geq 1$, since $\left|\xi_{2}\right| \gg\left|\xi_{3}\right|$ and $|\xi| \leq C\left|\xi_{2}\right|$, we have

$$
\begin{aligned}
K_{1}\left(\xi_{1}, \tau_{1}, \xi_{2}, \tau_{2}, \xi, \tau\right) & \leq C \frac{\left|\xi_{2}\right|\left|\xi_{1}\right|\langle\xi\rangle^{s}}{\langle\sigma\rangle^{-b^{\prime}}\left(1+\xi^{2}\right) \prod_{j=1}^{3}\left\langle\xi_{j}\right\rangle^{s}\left\langle\sigma_{j}\right\rangle^{b}} \\
& \leq \frac{C}{\langle\sigma\rangle^{-b^{\prime}} \prod_{j=1}^{3}\left\langle\sigma_{j}\right\rangle^{b}} .
\end{aligned}
$$

This case can be treated similarly to $J_{1}$.

When $1 / 4 \leq s \leq 1$, we consider the case $\left|\xi_{1}+\xi_{2}\right| \leq\left|\xi_{3}\right| / 3$ and the case $\left|\xi_{1}+\xi_{2}\right| \geq\left|\xi_{3}\right| / 3$.

When $\left|\xi_{1}+\xi_{2}\right| \leq\left|\xi_{3}\right| / 3$, since $1 / 4 \leq s \leq 1$, we have

$$
\begin{aligned}
K_{1}\left(\xi_{1}, \xi_{2}, \xi, \tau_{1}, \tau_{2}, \tau\right) & \leq C \frac{\left|\xi_{2}\right|^{2-2 s}}{\langle\sigma\rangle^{-b^{\prime}} \prod_{j=1}^{3}\left\langle\sigma_{j}\right\rangle^{b}} \\
& \leq C \frac{\left|\xi_{2}\right|^{3 / 2}}{\langle\sigma\rangle^{-b^{\prime}} \prod_{j=1}^{3}\left\langle\sigma_{j}\right\rangle^{b}} .
\end{aligned}
$$

This case can be treated similarly to $J_{2}$.

When $\left|\xi_{1}+\xi_{2}\right| \geq\left|\xi_{3}\right| / 3$, From Lemma 5, we have

$$
\begin{aligned}
\left|\sigma-\left(\sum_{j=1}^{3} \sigma_{j}\right)\right| & =\left|\xi^{3}-\xi_{1}^{3}-\xi_{2}^{3}-\xi_{3}^{3}\right| \\
& =3\left|\left(\xi_{1}+\xi_{2}\right)\left(\xi_{1}+\xi_{3}\right)\left(\xi_{2}+\xi_{3}\right)\right| \geq C\left|\xi_{1}\right|^{2}\left|\xi_{3}\right|,
\end{aligned}
$$

which yields $\max \left\{|\sigma|,\left|\sigma_{1}\right|,\left|\sigma_{2}\right|,\left|\sigma_{3}\right|\right\} \geq C\left|\xi_{1}\right|^{2}\left|\xi_{3}\right|$; thus one of the following four cases must occur:

$$
\begin{aligned}
& |\sigma|=\max \left\{|\sigma|,\left|\sigma_{1}\right|,\left|\sigma_{2}\right|,\left|\sigma_{3}\right|\right\} \geq C\left|\xi_{1}\right|^{2}\left|\xi_{3}\right|, \\
& \left|\sigma_{1}\right|=\max \left\{|\sigma|,\left|\sigma_{1}\right|,\left|\sigma_{2}\right|,\left|\sigma_{3}\right|\right\} \geq C\left|\xi_{1}\right|^{2}\left|\xi_{3}\right|, \\
& \left|\sigma_{2}\right|=\max \left\{|\sigma|,\left|\sigma_{1}\right|,\left|\sigma_{2}\right|,\left|\sigma_{3}\right|\right\} \geq C\left|\xi_{1}\right|^{2}\left|\xi_{3}\right|, \\
& \left|\sigma_{3}\right|=\max \left\{|\sigma|,\left|\sigma_{1}\right|,\left|\sigma_{2}\right|,\left|\sigma_{3}\right|\right\} \geq C\left|\xi_{1}\right|^{2}\left|\xi_{3}\right| .
\end{aligned}
$$

When (37) is valid, since

$$
\frac{|\xi|\langle\xi\rangle^{s}}{1+\xi^{2}} \leq 1
$$

we have

$$
\begin{aligned}
K_{1}\left(\xi_{1}, \tau_{1}, \xi_{2}, \tau_{2}, \xi, \tau\right) & \leq C \frac{\left|\xi_{2}\right|\left|\xi_{3}\right|}{\langle\sigma\rangle^{-b^{\prime}} \prod_{j=1}^{3}\left\langle\xi_{j}\right\rangle^{s}\left\langle\sigma_{j}\right\rangle^{b}} \\
& \leq C \frac{\left|\xi_{2}\right|^{1-2 s}\left|\xi_{3}\right|^{1-s}}{\langle\sigma\rangle^{-b^{\prime}} \prod_{j=1}^{3}\left\langle\sigma_{j}\right\rangle^{b}} \\
& \leq C \frac{\left|\xi_{2}\right|^{1-2 s+2 b^{\prime}}\left|\xi_{3}\right|^{1-s+b^{\prime}}}{\prod_{j=1}^{3}\left\langle\sigma_{j}\right\rangle^{b}} ;
\end{aligned}
$$

when $1-s+b^{\prime} \leq 0$, since $1 / 4 \leq s \leq 1$ and $b^{\prime}=-1 / 2+3 \epsilon$, we have

$$
K_{1}\left(\xi_{1}, \tau_{1}, \xi_{2}, \tau_{2}, \xi, \tau\right) \leq C \frac{\left|\xi_{2}\right|^{1-2 s+2 b^{\prime}}}{\prod_{j=1}^{3}\left\langle\sigma_{j}\right\rangle^{b}} \leq \frac{C}{\prod_{j=1}^{3}\left\langle\sigma_{j}\right\rangle^{b}} ;
$$

when $1-s+b^{\prime} \geq 0$, since $1 / 4 \leq s \leq 1$ and $b^{\prime}=-1 / 2+3 \epsilon$, we have

$$
K_{1}\left(\xi_{1}, \tau_{1}, \xi_{2}, \tau_{2}, \xi, \tau\right) \leq C \frac{\left|\xi_{2}\right|^{2-3 s+3 b^{\prime}}}{\prod_{j=1}^{3}\left\langle\sigma_{j}\right\rangle^{b}} \leq \frac{C}{\prod_{j=1}^{3}\left\langle\sigma_{j}\right\rangle^{b}} .
$$

By using Cauchy-Schwartz's inequality and Plancherel's identity, (11), $8 b / 9<b$, we obtain

$$
\begin{aligned}
& J_{4} \leq C \int_{\mathbf{R}^{2}} \int_{\substack{\xi=\xi_{1}+\xi_{2}+\xi_{3} \\
\tau=\tau_{1}+\tau_{2}+\tau_{3}}} \frac{F \prod_{j=1}^{3} F_{j}}{\prod_{j=1}^{3}\left\langle\sigma_{j}\right\rangle^{b}} d \xi_{1} d \tau_{1} d \xi_{2} d \tau_{2} d \xi d \tau \\
& \leq C\|F\|_{L_{\xi \tau}^{2}} \prod_{j=1}^{3}\left\|f_{j}\right\|_{L_{x t}^{6}} \\
& \leq C\|F\|_{L_{\xi \tau}^{2}} \prod_{j=1}^{3}\left\|f_{j}\right\|_{X_{0,8 b / 9}} \\
& \leq C\|F\|_{L_{\xi_{\tau}}^{2}} \prod_{j=1}^{3}\left\|F_{j}\right\|_{L_{\xi_{\tau}}^{2}} .
\end{aligned}
$$

When (38) is valid, since

$$
\frac{|\xi|\langle\xi\rangle^{s}}{1+\xi^{2}} \leq 1
$$

we have

$$
\begin{aligned}
K_{1}\left(\xi_{1}, \tau_{1}, \xi_{2}, \tau_{2}, \xi, \tau\right) & \leq C \frac{\left|\xi_{2}\right|\left|\xi_{3}\right|}{\langle\sigma\rangle^{-b^{\prime}} \prod_{j=1}^{3}\left\langle\xi_{j}\right\rangle^{s}\left\langle\sigma_{j}\right\rangle^{b}} \\
& \leq C \frac{\left|\xi_{2}\right|^{1-2 s}\left|\xi_{3}\right|^{1-s}}{\langle\sigma\rangle^{-b^{\prime}} \prod_{j=1}^{3}\left\langle\sigma_{j}\right\rangle^{b}} \\
& \leq C \frac{\left|\xi_{2}\right|^{1-2 s+2 b^{\prime}}\left|\xi_{3}\right|^{1-s+b^{\prime}}}{\langle\sigma\rangle^{-b^{\prime}} \prod_{j=2}^{3}\left\langle\sigma_{j}\right\rangle^{b}}
\end{aligned}
$$


when $1-s+b^{\prime} \leq 0$, we have

$$
\begin{aligned}
K_{1}\left(\xi_{1}, \tau_{1}, \xi_{2}, \tau_{2}, \xi, \tau\right) & \leq C \frac{\left|\xi_{2}\right|^{1-2 s+2 b^{\prime}}}{\langle\sigma\rangle^{-b^{\prime}} \prod_{j=2}^{3}\left\langle\sigma_{j}\right\rangle^{b}} \\
& \leq \frac{C}{\langle\sigma\rangle^{-b^{\prime}} \prod_{j=2}^{3}\left\langle\sigma_{j}\right\rangle^{b}} ;
\end{aligned}
$$

when $1-s+b^{\prime} \geq 0$, since $1 / 4 \leq s \leq 1$ and $b^{\prime}=-1 / 2+3 \epsilon$, we have

$$
\begin{aligned}
K_{1}\left(\xi_{1}, \tau_{1}, \xi_{2}, \tau_{2}, \xi, \tau\right) & \leq C \frac{\left|\xi_{2}\right|^{2-3 s+3 b^{\prime}}}{\langle\sigma\rangle^{-b^{\prime}} \prod_{j=2}^{3}\left\langle\sigma_{j}\right\rangle^{b}} \\
& \leq \frac{C}{\langle\sigma\rangle^{-b^{\prime}} \prod_{j=2}^{3}\left\langle\sigma_{j}\right\rangle^{b}} .
\end{aligned}
$$

By using Cauchy-Schwartz's inequality and Plancherel's identity, (11), $8 b / 9<b$, and $8 b / 9<-b^{\prime}$, we obtain

$$
\begin{aligned}
& J_{4} \leq C \int_{\mathbf{R}^{2}} \int_{\substack{\xi=\xi_{1}+\xi_{2}+\xi_{3} \\
\tau=\tau_{1}+\tau_{2}+\tau_{3}}} \frac{F \prod_{j=1}^{3} F_{j}}{\langle\sigma\rangle^{-b^{\prime}} \prod_{j=2}^{3}\left\langle\sigma_{j}\right\rangle^{b}} d \xi_{1} d \tau_{1} d \xi_{2} d \tau_{2} d \xi d \tau \\
& \leq C\left\|F_{1}\right\|_{L_{\xi_{\tau}}^{2}}\|f\|_{L_{x t}^{6}} \prod_{j=2}^{3}\left\|f_{j}\right\|_{L_{x t}^{6}} \\
& \leq C\left\|F_{1}\right\|_{L_{\xi_{\tau}}^{2}}\|f\|_{X_{0,8 b / 9}} \prod_{j=2}^{3}\left\|f_{j}\right\|_{X_{0,8 b / 9}} \\
& \leq C\|F\|_{L_{\xi_{\tau}}^{2}} \prod_{j=1}^{3}\left\|F_{j}\right\|_{L_{\xi_{\tau}}^{2}} .
\end{aligned}
$$

Cases (39) and (40) can be treated similarly to case (38).

(5) Subregion $\left\{\left|\xi_{1}\right| \geq 1,\left|\xi_{1}\right| \sim\left|\xi_{2}\right| \sim\left|\xi_{3}\right|,\left|\xi_{1}\right| \geq\left|\xi_{2}\right| \geq\right.$ $\left.\left|\xi_{3}\right|,|\xi| \geq\left|\xi_{3}\right| / 3\right\}$. In this subregion, since $s \geq 1 / 4,\left|\xi_{1}\right| \sim|\xi| \sim$ $\left|\xi_{2}\right| \sim\left|\xi_{3}\right|$ which yields

$$
\begin{aligned}
K_{1}\left(\xi_{1}, \tau_{1}, \xi_{2}, \tau_{2}, \xi, \tau\right) & \leq C \frac{\left|\xi_{1}\right|^{1-2 s}}{\langle\sigma\rangle^{-b^{\prime}} \prod_{j=1}^{3}\left\langle\sigma_{j}\right\rangle^{b}} \\
& \leq C \frac{\left|\xi_{1}\right|^{1 / 2}}{\langle\sigma\rangle^{-b^{\prime}} \prod_{j=1}^{3}\left\langle\sigma_{j}\right\rangle^{b}} .
\end{aligned}
$$

Thus, applying Cauchy-Schwartz's inequality and Plancherel's identity, (12) and (13), we have

$$
\begin{aligned}
J_{5} & \leq C \int_{\mathbf{R}^{2}} \int_{\substack{\xi=\xi_{1}+\xi_{2}+\xi_{3} \\
\tau=\tau_{1}+\tau_{2}+\tau_{3}}} \frac{\left|\xi_{1}\right|^{1 / 2} F \prod_{j=1}^{3} F_{j}}{\langle\sigma\rangle^{-b^{\prime}} \prod_{j=1}^{3}\left\langle\sigma_{j}\right\rangle^{b}} d \xi_{1} d \tau_{1} d \xi_{2} d \tau_{2} d \xi d \tau \\
& \leq C\left\|D_{x} f_{1}\right\|_{L_{x}^{\infty} L_{t}^{2}}\left\|D_{x}^{-1 / 4} f_{2}\right\|_{L_{x}^{4} L_{t}^{\infty}}\left\|D_{x}^{-1 / 4} f_{3}\right\|_{L_{x}^{4} L_{t}^{\infty}}\|f\|_{L_{x t}^{2}}
\end{aligned}
$$

$$
\begin{aligned}
& \leq C\|F\|_{L_{\xi \tau}^{2}} \prod_{j=1}^{3}\left\|f_{j}\right\|_{X_{0, b}} \\
& \leq C\|F\|_{L_{\xi_{\tau}}^{2}} \prod_{j=1}^{3}\left\|F_{j}\right\|_{L_{\xi \tau}^{2}} .
\end{aligned}
$$

(6) Subregion $\left\{\left|\xi_{1}\right| \geq 1,\left|\xi_{1}\right| \sim\left|\xi_{2}\right| \sim\left|\xi_{3}\right|,\left|\xi_{1}\right| \geq\left|\xi_{2}\right| \geq\right.$ $\left.\left|\xi_{3}\right|,|\xi| \leq\left|\xi_{3}\right| / 3\right\}$. In this subregion, since $\left|\xi_{1}\right| \geq\left|\xi_{2}\right| \geq\left|\xi_{3}\right| \geq$

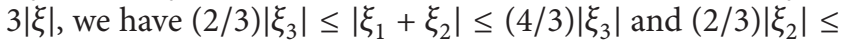
$\left|\xi_{1}+\xi_{3}\right| \leq(4 / 3)\left|\xi_{2}\right|$ and $(2 / 3)\left|\xi_{1}\right| \leq\left|\xi_{2}+\xi_{3}\right| \leq(4 / 3)\left|\xi_{1}\right|$. From Lemma 5 , we have

$$
\begin{aligned}
\mid \sigma & -\left(\sum_{j=1}^{3} \sigma_{j}\right) \mid \\
& =\left|\xi^{3}-\xi_{1}^{3}-\xi_{2}^{3}-\xi_{3}^{3}\right| \\
& =3\left|\left(\xi_{1}+\xi_{2}\right)\left(\xi_{1}+\xi_{3}\right)\left(\xi_{2}+\xi_{3}\right)\right| \sim\left|\xi_{1}\right|^{3} \sim\left|\xi_{2}\right|^{3} \sim\left|\xi_{3}\right|^{3}
\end{aligned}
$$

which yields $\max \left\{|\sigma|,\left|\sigma_{1}\right|,\left|\sigma_{2}\right|,\left|\sigma_{3}\right|\right\} \geq C\left|\xi_{1}\right|^{3}$; thus one of the following four cases must occur:

$$
\begin{gathered}
|\sigma|=\max \left\{|\sigma|,\left|\sigma_{1}\right|,\left|\sigma_{2}\right|,\left|\sigma_{3}\right|\right\} \geq C\left|\xi_{1}\right|^{3}, \\
\left|\sigma_{1}\right|=\max \left\{|\sigma|,\left|\sigma_{1}\right|,\left|\sigma_{2}\right|,\left|\sigma_{3}\right|\right\} \geq C\left|\xi_{1}\right|^{3}, \\
\left|\sigma_{2}\right|=\max \left\{|\sigma|,\left|\sigma_{1}\right|,\left|\sigma_{2}\right|,\left|\sigma_{3}\right|\right\} \geq C\left|\xi_{1}\right|^{3}, \\
\left|\sigma_{3}\right|=\max \left\{|\sigma|,\left|\sigma_{1}\right|,\left|\sigma_{2}\right|,\left|\sigma_{3}\right|\right\} \geq C\left|\xi_{1}\right|^{3} .
\end{gathered}
$$

When $s \geq 1$, we have

$$
\begin{aligned}
K_{1}\left(\xi_{1}, \tau_{1}, \tau_{2}, \xi_{2}, \xi, \tau\right) & \leq C \frac{\left|\xi_{1}\right|^{2-2 s}}{\langle\sigma\rangle^{-b^{\prime}} \prod_{j=1}^{3}\left\langle\sigma_{j}\right\rangle^{b}} \\
& \leq \frac{C}{\langle\sigma\rangle^{-b^{\prime}} \prod_{j=1}^{3}\left\langle\sigma_{j}\right\rangle^{b}} ;
\end{aligned}
$$

this case can be handled similarly to $J_{1}$.

When $1 / 4 \leq s \leq 1$, we consider (54), (55), (56), and (57), respectively.

When (37) is valid, since $\left|\xi_{1}\right| \sim\left|\xi_{2}\right| \sim\left|\xi_{3}\right|,|\xi| \leq\left|\xi_{3}\right|$ and $b^{\prime}=-1 / 2+3 \epsilon$ as well as $1 / 4 \leq s \leq 1$, we have

$$
\begin{aligned}
K_{1}\left(\xi_{1}, \tau_{1}, \xi_{2}, \tau_{2}, \xi, \tau\right) & \leq C \frac{|\xi|\langle\xi\rangle^{s}\left|\xi_{1}\right|^{2-3 s}}{\left(1+\xi^{2}\right)\langle\sigma\rangle^{-b^{\prime}} \prod_{j=1}^{3}\left\langle\sigma_{j}\right\rangle^{b}} \\
& \leq C \frac{\left|\xi_{1}\right|^{2-3 s+3 b^{\prime}}}{\prod_{j=1}^{3}\left\langle\sigma_{j}\right\rangle^{b}} \leq C \frac{\left|\xi_{1}\right|^{9 \epsilon-(1 / 4)}}{\prod_{j=1}^{3}\left\langle\sigma_{j}\right\rangle^{b}} \\
& \leq \frac{C}{\prod_{j=1}^{3}\left\langle\sigma_{j}\right\rangle^{b}} .
\end{aligned}
$$


By using Cauchy-Schwartz's inequality and Plancherel's identity, (11), $8 b / 9<b$, we obtain

$$
\begin{aligned}
& J_{6} \leq C \int_{\mathbf{R}^{2}} \int_{\substack{\xi=\xi_{1}+\xi_{2}+\xi_{3} \\
\tau=\tau_{1}+\tau_{2}+\tau_{3}}} \frac{F \prod_{j=1}^{3} F_{j}}{\prod_{j=1}^{3}\left\langle\sigma_{j}\right\rangle^{b}} d \xi_{1} \mathrm{~d} \tau_{1} d \xi_{2} d \tau_{2} d \xi d \tau \\
& \leq C\|F\|_{L_{\xi \tau}^{2}} \prod_{j=1}^{3}\left\|f_{j}\right\|_{L_{x t}^{6}} \\
& \leq C\|F\|_{L_{\xi \tau}^{2}} \prod_{j=1}^{3}\left\|f_{j}\right\|_{X_{0,8 b / 9}} \\
& \leq C\|F\|_{L_{\xi \tau}^{2}} \prod_{j=1}^{3}\left\|F_{j}\right\|_{L_{\xi \tau}^{2}} .
\end{aligned}
$$

When (55) holds, since $\left|\xi_{1}\right| \sim\left|\xi_{2}\right| \sim\left|\xi_{3}\right|,|\xi| \leq\left|\xi_{3}\right|$ and $b^{\prime}=$ $-1 / 2+3 \epsilon$ as well as $1 / 4 \leq s \leq 1$, we have

$$
\begin{aligned}
K_{1}\left(\xi_{1}, \tau_{1}, \xi_{2}, \tau_{2}, \xi, \tau\right) & \leq C \frac{|\xi|\langle\xi\rangle^{s}\left|\xi_{1}\right|^{2-3 s}}{\left(1+\xi^{2}\right)\langle\sigma\rangle^{-b^{\prime}} \prod_{j=1}^{3}\left\langle\sigma_{j}\right\rangle^{b}} \\
& \leq \frac{\left|\xi_{1}\right|^{2-3 s+3 b^{\prime}}}{\langle\sigma\rangle^{-b^{\prime}} \prod_{j=2}^{3}\left\langle\sigma_{j}\right\rangle^{b}} \\
& \leq C \frac{\left|\xi_{1}\right|^{9 \epsilon-(1 / 4)}}{\langle\sigma\rangle^{-b^{\prime}} \prod_{j=2}^{3}\left\langle\sigma_{j}\right\rangle^{b}} \\
& \leq \frac{C^{-b^{\prime}} \prod_{j=2}^{3}\left\langle\sigma_{j}\right\rangle^{b}}{\langle\sigma\rangle^{2}}
\end{aligned}
$$

By using Cauchy-Schwartz's inequality and Plancherel's identity, (11), $8 b / 9<b$, and $8 b / 9<-b^{\prime}$, we obtain

$$
\begin{aligned}
J_{6} & \leq C \int_{\mathbf{R}^{2}} \int_{\substack{\xi=\xi_{1}+\xi_{2}+\xi_{3} \\
\tau=\tau_{1}+\tau_{2}+\tau_{3}}} \frac{F \prod_{j=1}^{3} F_{j}}{\langle\sigma\rangle^{-b^{\prime}} \prod_{j=2}^{3}\left\langle\sigma_{j}\right\rangle^{b}} d \xi_{1} d \tau_{1} d \xi_{2} d \tau_{2} d \xi d \tau \\
& \leq C\left\|F_{1}\right\|_{L_{\xi_{\tau}}^{2}}\|f\|_{L_{x t}^{6}} \prod_{j=2}^{3}\left\|f_{j}\right\|_{L_{x t}^{6}} \\
& \leq C\left\|F_{1}\right\|_{L_{\xi \tau}^{2}}\|f\|_{X_{0,8 b / 9}} \prod_{j=2}^{3}\left\|f_{j}\right\|_{X_{0,8 b / 9}} \\
& \leq C\|F\|_{L_{\xi \tau}^{2}} \prod_{j=1}^{3}\left\|F_{j}\right\|_{L_{\xi_{\tau}}^{2}} \cdot
\end{aligned}
$$

Cases (56) and (57) can be treated similarly to case (55).

Putting the estimates of $J_{k}(1 \leq k \leq 6, k \in \mathrm{Z})$ together, we have (25).

Thus, we complete the proof of Lemma 7.
Lemma 8. Let $s \geq 1 / 4, b=1 / 2+2 \epsilon, b^{\prime}=-1 / 2+3 \epsilon, 0<\epsilon \ll 1$. Then

$$
\left\|\partial_{x}\left(u_{1} u_{2} u_{3}\right)\right\|_{X_{s, b^{\prime}}} \leq C \prod_{j=1}^{3}\left\|u_{j}\right\|_{X_{s, b}} .
$$

Lemma 8 can be proved similarly to Lemma 7 .

\section{The Proof of Theorem 1}

In order to prove Theorem 1, firstly, for $\phi \in H^{s}(\mathbf{R})$ and $\delta \in$ $(0,1], u \in X_{s, b}\left(\mathbf{R}^{2}\right)$, we define $G_{\phi}(u)$ by

$$
G_{\phi}(v)=\psi(t) W(t) \phi-\psi_{\delta} \int_{0}^{t} W\left(t-t^{\prime}\right) g\left(u, \partial_{x} u\right) d t^{\prime}
$$

where

$$
g\left(u, \partial_{x} u\right)=\frac{1}{3} \partial_{x}\left(u^{3}\right)+\left(1-\partial_{x}^{2}\right)^{-1} \partial_{x}\left[\frac{5}{3} u^{3}+u\left(\partial_{x} u\right)^{2}\right] .
$$

By using Lemmas 4, 7, and 8, we have

$$
\left\|G_{\phi}(u)\right\|_{X_{s, b}\left(\mathbf{R}^{2}\right)} \leq C\|\phi\|_{H^{s}(\mathbf{R})}+C \delta^{b^{\prime}+1-b}\|u\|_{X_{s, b}\left(\mathbf{R}^{2}\right)}^{3},
$$

where $s, b, b^{\prime}$ of (66) concord with $s, b$, and $b^{\prime}$ of Lemmas 7 and 8 . Let

$$
\begin{aligned}
& \delta=\left(\frac{1}{16 C^{3}\left(\|\phi\|_{H^{s}(\mathbf{R})}^{2}+2\right)}\right)^{1 /\left(b^{\prime}+1-b\right)}, \\
& r=2 C\|\phi\|_{H^{s}(\mathbf{R})}
\end{aligned}
$$

where $0<\delta<1$. From (66) and (67), we know that $G_{\phi}$ is a mapping from the closed ball $B(0, r)=\left\{u \in X_{s, b}\left(\mathbf{R}^{2}\right)\right.$, $\left.\|u\|_{X_{s, b\left(\mathbf{R}^{2}\right)}} \leq r\right\}$ into itself. Similarly, we have

$$
\begin{aligned}
& \left\|G_{\phi}(v)-G_{\phi}(u)\right\|_{X_{s, b}\left(\mathbf{R}^{2}\right)} \\
& \quad \leq 2 C \delta^{b^{\prime}+1-b}\|v-u\|_{X_{s, b}\left(\mathbf{R}^{2}\right)}\left(\|u\|_{X_{s, b}\left(\mathbf{R}^{2}\right)}^{2}+\|v\|_{X_{s, b}\left(\mathbf{R}^{2}\right)}^{2}\right) \\
& \quad \leq \frac{1}{2}\|v-u\|_{X_{s, b}\left(\mathbf{R}^{2}\right)}
\end{aligned}
$$

thus, $G_{\phi}$ is a contraction mapping from the closed ball $B(0, r)=\left\{u \in X_{s, b}\left(\mathbf{R}^{2}\right),\|u\|_{X_{s, b}\left(\mathbf{R}^{2}\right)} \leq r\right\}$ into itself; by using Banach fixed point Theorem, we have $G_{\phi}(u)=u$.

The rest of local well-posedness of Theorem 1 follows from a standard proof.

\section{The Proof of Theorem 2}

In this section, we prove Theorem 2.

By contradiction, we assume that the solution map of (1) and (2)

$$
S_{t}: u_{0} \in H^{s}(\mathbf{R}) \longrightarrow u \in C\left([0, T] ; H^{s}(\mathbf{R})\right)
$$


is Lipschitz continuous at zero with $s<1 / 4$. From the general well-posedness principle of [20], we must have

$$
\sup _{t \in[0, T]}\left\|B_{3}\left(u_{0}\right)\right\|_{H^{s}} \leq C\left\|u_{0}\right\|_{H^{s}}^{3}, \quad \forall u_{0} \in H^{s}(\mathbf{R}),
$$

where

$$
\begin{gathered}
B_{3}\left(u_{0}\right)(x, t) \\
=\int_{0}^{t} W(t-\tau) \partial_{x}\left(B_{1}\left(u_{0}\right) B_{1}\left(u_{0}\right) B_{1}\left(u_{0}\right)\right)(\tau) d \tau, \\
B_{1}\left(u_{0}\right)(x, t)=W(t) u_{0} .
\end{gathered}
$$

We consider the initial data

$$
u_{0}(x)=r^{-1 / 2} N^{-s}\left\{e^{-i N x}\left(\int_{0}^{r} e^{i x \xi} d \xi\right)+e^{i N x}\left(\int_{r}^{2 r} e^{i x \xi} d \xi\right)\right\},
$$

where $r^{2} N=O(1)$ and $N \gg 1$. Thus, we have

$$
\mathscr{F}_{x} u_{0}(\xi)=C r^{-1 / 2} N^{-s}\left\{\chi_{[-N,-N+r]}(\xi)+\chi_{[N+r, N+2 r]}(\xi)\right\},
$$

which can be seen in [24], where $\chi_{I}$ denotes the characteristic function of a set $I \subset \mathbf{R}$. It is easy to check that $\left\|u_{0}\right\|_{H^{s}} \sim 1$. Let $I_{1}=[-N,-N+r]$ and $I_{2}=[N+r, N+2 r]$ and $I_{1} \cup I_{2}=\Omega$. We have

$$
\mathscr{F}_{x} B_{1}\left(u_{0}\right)(\xi)=C e^{i t \phi(\xi)} \mathscr{F}_{x} u_{0}(\xi)
$$

and thus

$$
\begin{aligned}
& B_{1}\left(u_{0}\right)(x, t)=C r^{-1 / 2} N^{-s} \int_{\xi \in \Omega} e^{i x \xi} e^{i t \phi(\xi)} d \xi \\
& B_{3}\left(u_{0}\right)(x, t)=C f
\end{aligned}
$$

where

$$
\begin{aligned}
f=r^{-3 / 2} N^{-3 s} \int_{\xi_{1} \in \Omega} \int_{\xi_{2} \in \Omega} \int_{\xi_{3} \in \Omega} & \frac{\xi}{3\left(1+\xi^{2}\right)} \\
& \times\left(\xi^{2}+6-3 \xi_{1} \xi_{2}\right) \\
& \times e^{i x\left(\xi_{1}+\xi_{2}+\xi_{3}\right)} Q d \xi_{1} d \xi_{2} d \xi_{3},
\end{aligned}
$$

where

$$
Q=\frac{e^{i t\left(\phi\left(\xi_{1}\right)+\phi\left(\xi_{2}\right)+\phi\left(\xi_{3}\right)\right)}-e^{i t \phi\left(\xi_{1}+\xi_{2}+\xi_{3}\right)}}{\phi\left(\xi_{1}\right)+\phi\left(\xi_{2}\right)+\phi\left(\xi_{3}\right)-\phi\left(\xi_{1}+\xi_{2}+\xi_{3}\right)} .
$$

Since $\phi(\xi)=-\xi^{3}$, we define

$$
\begin{aligned}
\theta & :=\phi\left(\xi_{1}\right)+\phi\left(\xi_{2}\right)+\phi\left(\xi_{3}\right)-\phi\left(\xi_{1}+\xi_{2}+\xi_{3}\right) \\
& =3\left(\xi_{1}+\xi_{2}\right)\left(\xi_{1}+\xi_{3}\right)\left(\xi_{2}+\xi_{3}\right)
\end{aligned}
$$

resulting from Lemma 5 and $\xi=\xi_{1}+\xi_{2}+\xi_{3}$. To estimate $\|f\|_{H^{s}}$, we consider the following three cases.
Case $1: \xi_{j}(j=1,2,3) \in I_{1}$.

Case $2: \xi_{j}(j=1,2,3) \in I_{2}$.

Case 3: $\xi_{j}(j=1,2) \in I_{1}, \xi_{3} \in I_{2}$, or $\xi_{1} \in I_{1}, \xi_{j}(j=$ $2,3) \in I_{2}$, or $\xi_{j}(j=1,2) \in I_{2}, \xi_{3} \in I_{1}$, or $\xi_{1} \in I_{2}$, $\xi_{j}(j=2,3) \in I_{1}$.

The integrals in (76) corresponding to Cases 1, 2, and 3 are denoted as $f_{1}, f_{2}, f_{3}$, respectively.

Case 1. In this case, from Lemma 6, we have $|\theta| \sim N^{3}$ and $\left|\xi^{2}+6-3 \xi_{1} \xi_{2}\right| \sim N^{2}$. Since $r^{2} N=O(1)$, we have

$$
\left\|f_{1}\right\|_{H^{s}} \leq C r^{-3 / 2} N^{-3 s} N^{s} r^{5 / 2} N^{-2} \sim N^{-2 s-5 / 2} .
$$

Case 2. In this case, from Lemma 6, we have $|\theta| \sim N^{3}$ and $\left|\xi^{2}+6-3 \xi_{1} \xi_{2}\right| \sim N^{2}$. Since $r^{2} N=O(1)$, we have

$$
\left\|f_{2}\right\|_{H^{s}} \leq C r^{-3 / 2} N^{-3 s} N^{s} r^{5 / 2} N^{-2} \sim N^{-2 s-5 / 2} .
$$

Case 3. In this case $|\theta| \sim r^{2} N=O(1),|Q| \geq$ Constant due to Lemma 6 , and $\left|\xi^{2}+6-3 \xi_{1} \xi_{2}\right| \sim N^{2}$. Since $r^{2} N=O(1)$, we have

$$
\left\|f_{3}\right\|_{H^{s}} \geq C r^{-3 / 2} N^{-3 s} N^{s} r^{5 / 2} N \sim N^{-2 s+1 / 2} .
$$

From (70), we have

$$
\begin{aligned}
C \sim C\left\|u_{0}\right\|_{H^{s}}^{3} & \geq\left\|f_{1}+f_{2}+f_{3}\right\|_{H^{s}} \\
& \geq\left\|f_{3}+f_{2}\right\|_{H^{s}}-\left\|f_{1}\right\|_{H^{s}} \\
& \geq\left\|f_{3}\right\|_{H^{s}}-\left\|f_{2}\right\|_{H^{s}}-\left\|f_{1}\right\|_{H^{s}} \\
& \geq C\left(N^{-2 s+1 / 2}-N^{-2 s-5 / 2}\right)
\end{aligned}
$$

which yields

$$
N^{-2 s+1 / 2} \leq C\left(1+N^{-2 s-5 / 2}\right)
$$

When $s<-5 / 4$, from (83), we have $N^{-2 s+1 / 2} \leq C N^{-2 s-5 / 2}$ which yields $N^{3} \leq C$; we obtain a contradiction since $N \gg 1$. When $-5 / 4 \leq s<1 / 4$, from (83), we have $N^{-2 s+1 / 2} \leq C$; we obtain a contradiction since $N \gg 1$.

The proof of Theorem 2 is completed.

\section{Conflict of Interests}

The authors declare that there is no conflict of interests regarding the publication of this paper.

\section{Acknowledgments}

The research of the first author is supported by Foundation and Frontiers of Henan province under nos. 122300410414 and 132300410432 . 


\section{References}

[1] S. Hakkaev and K. Kirchev, "Local well-posedness and orbital stability of solitary wave solutions for the generalized CamassaHolm equation," Communications in Partial Differential Equations, vol. 30, no. 4-6, pp. 761-781, 2005.

[2] X. F. Liu and Y. Y. Jin, "The Cauchy problem of a shallow water equation," Acta Mathematica Sinica, vol. 21, no. 2, pp. 393-408, 2005.

[3] Y. Li, W. Yan, and X. Yang, "Well-posedness of a higher order modified Camassa-Holm equation in spaces of low regularity," Journal of Evolution Equations, vol. 10, no. 2, pp. 465-486, 2010.

[4] E. A. Olson, "Well posedness for a higher order modified Camassa-Holm equation," Journal of Differential Equations, vol. 246, no. 10, pp. 4154-4172, 2009.

[5] H. Wang and S. Cui, "Global well-posedness of the Cauchy problem of the fifth-order shallow water equation," Journal of Differential Equations, vol. 230, no. 2, pp. 600-613, 2006.

[6] A. Grunrock, New applications of the fourier restriction norm method to well-posedness problems for nonlinear evolution equations, [Doctoral Dissertation], University of Wuppertal, 2002.

[7] A. Grünrock, "An improved local well-posedness result for the modified KdV equation," International Mathematics Research Notices, no. 61, pp. 3287-3308, 2004.

[8] C. E. Kenig, G. Ponce, and L. Vega, "Well-posedness and scattering results for the generalized Korteweg-de Vries equation via the contraction principle," Communications on Pure and Applied Mathematics, vol. 46, no. 4, pp. 527-620, 1993.

[9] C. E. Kenig, G. Ponce, and L. Vega, "On the ill-posedness of some canonical dispersive equations," Duke Mathematical Journal, vol. 106, no. 3, pp. 617-633, 2001.

[10] C. E. Kenig, G. Ponce, and L. Vega, "A bilinear estimate with applications to the KdV equation," Journal of the American Mathematical Society, vol. 9, no. 2, pp. 573-603, 1996.

[11] J. Colliander, M. Keel, G. Staffilani, H. Takaoka, and T. Tao, "Sharp global well-posedness for KdV and modified $\mathrm{KdV}$ on $R$ and T," Journal of the American Mathematical Society, vol. 16, no. 3, pp. 705-749, 2003.

[12] J. Colliander, M. Keel, G. Staffilani, H. Takaoka, and T. Tao, "Global well-posedness for Schrödinger equations with derivative," SIAM Journal on Mathematical Analysis, vol. 33, no. 3, pp. 649-669, 2001.

[13] J. Colliander, M. Keel, G. Staffilani, H. Takaoka, and T. Tao, "Global well-posedness for KdV in Sobolev spaces of negative index," Electronic Journal of Differential Equations, vol. 2001, no. 26, pp. 1-7, 2001.

[14] J. Colliander, M. Keel, G. Staffilani, H. Takaoka, and T. Tao, "Almost conservation laws and global rough solutions to a nonlinear Schrödinger equation," Mathematical Research Letters, vol. 9, no. 5-6, pp. 659-682, 2002.

[15] R. M. Miura, "The Korteweg-de Vries equation: a survey of results," SIAM Review, vol. 18, no. 3, pp. 412-459, 1976.

[16] Z. Guo, "Global well-posedness of Korteweg-de Vries equation in $H^{-3 / 4}(R)$," Journal de Mathématiques Pures et Appliquées, vol. 91, no. 6, pp. 583-597, 2009.

[17] A. D. Ionescu and C. E. Kenig, "Global well-posedness of the Benjamin-Ono equation in low-regularity spaces," Journal of the American Mathematical Society, vol. 20, no. 3, p. 753-798 (electronic), 2007.

[18] A. D. Ionescu, C. E. Kenig, and D. Tataru, "Global well-posedness of the KP-I initial-value problem in the energy space," Inventiones Mathematicae, vol. 173, no. 2, pp. 265-304, 2008.
[19] J. Bourgain, "Fourier transform restriction phenomena for certain lattice subsets and applications to nonlinear evolution equations. II. The KdV-equation," Geometric and Functional Analysis, vol. 3, no. 3, pp. 209-262, 1993.

[20] I. Bejenaru and T. Tao, "Sharp well-posedness and ill-posedness results for a quadratic non-linear Schrödinger equation," Journal of Functional Analysis, vol. 233, no. 1, pp. 228-259, 2006.

[21] C. E. Kenig, G. Ponce, and L. Vega, "The Cauchy problem for the Korteweg-de Vries equation in Sobolev spaces of negative indices,” Duke Mathematical Journal, vol. 71, no. 1, pp. 1-21, 1993.

[22] C. E. Kenig, G. Ponce, and L. Vega, "Oscillatory integrals and regularity of dispersive equations," Indiana University Mathematics Journal, vol. 40, no. 1, pp. 33-69, 1991.

[23] J. Ginibre, "Le problème de Cauchy pour des EDP semilinéaires périodiques en variables d'espace (d'après Bourgain)," Astérisque, no. 237, pp. 163-187, 1996.

[24] N. Tzvetkov, "Remark on the local ill-posedness for KdV equation," Comptes Rendus de l'Académie des Sciences. Série I. Mathématique, vol. 329, no. 12, pp. 1043-1047, 1999. 


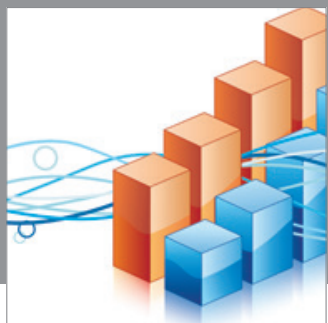

Advances in

Operations Research

mansans

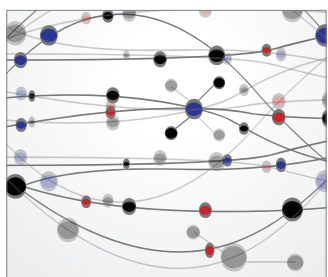

The Scientific World Journal
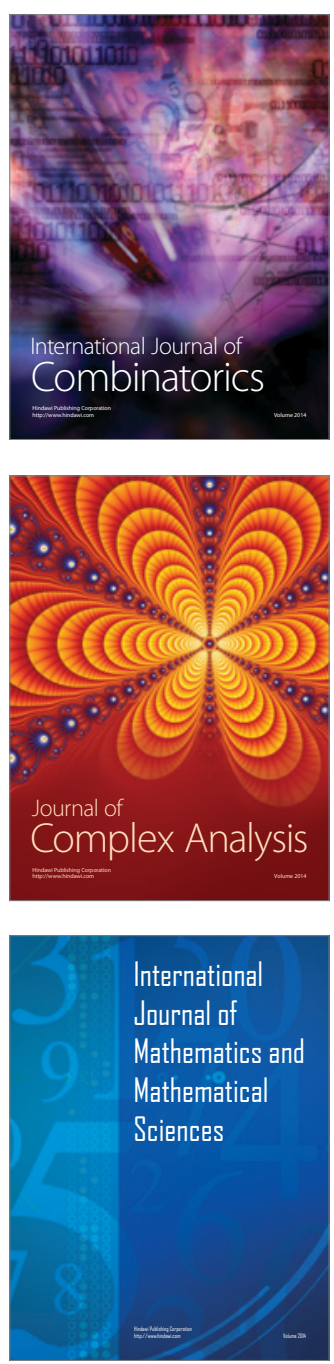
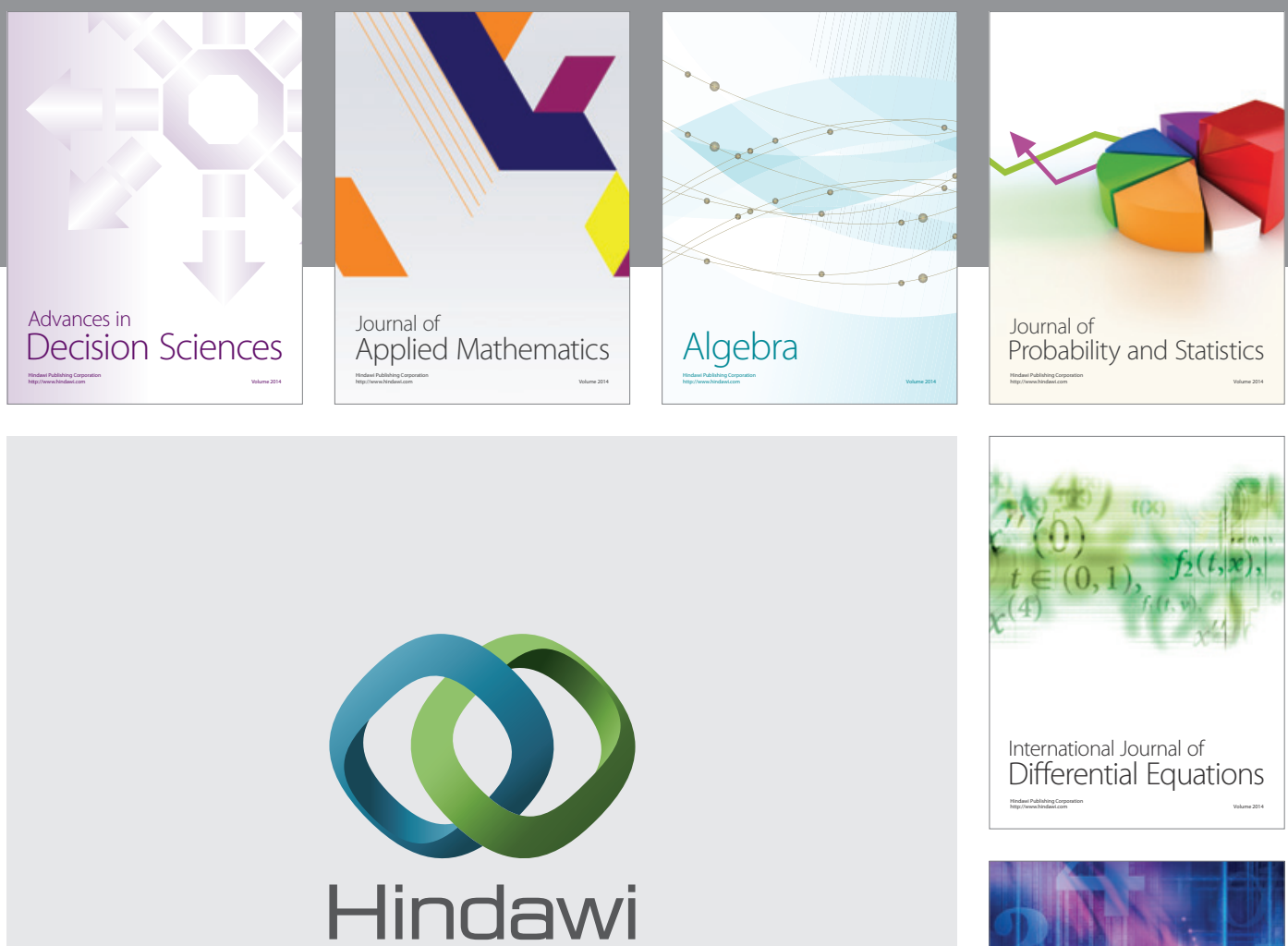

Submit your manuscripts at http://www.hindawi.com
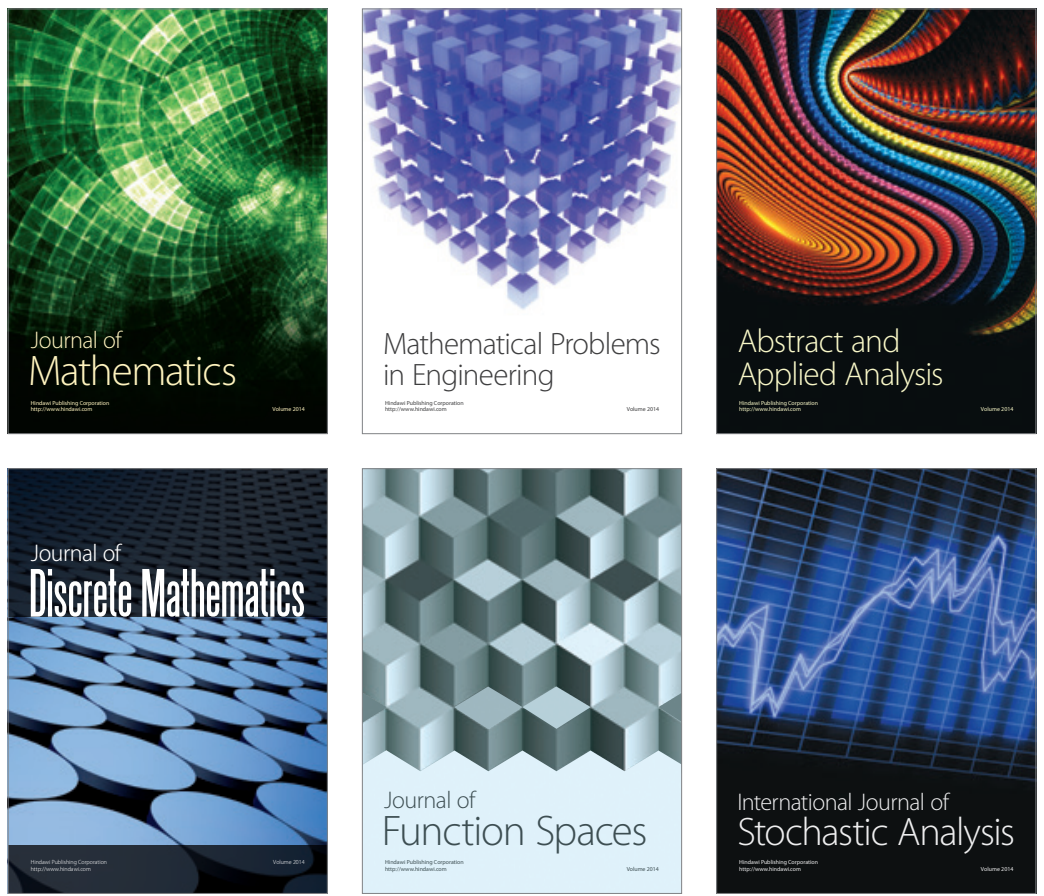

Journal of

Function Spaces

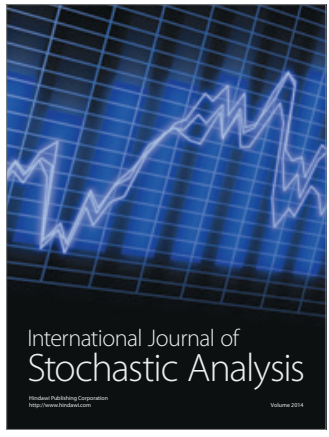

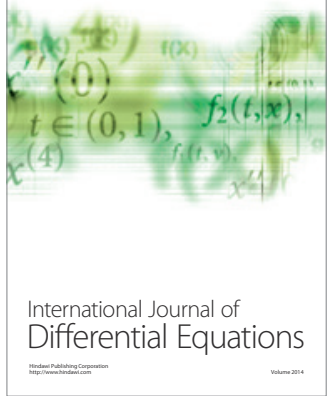
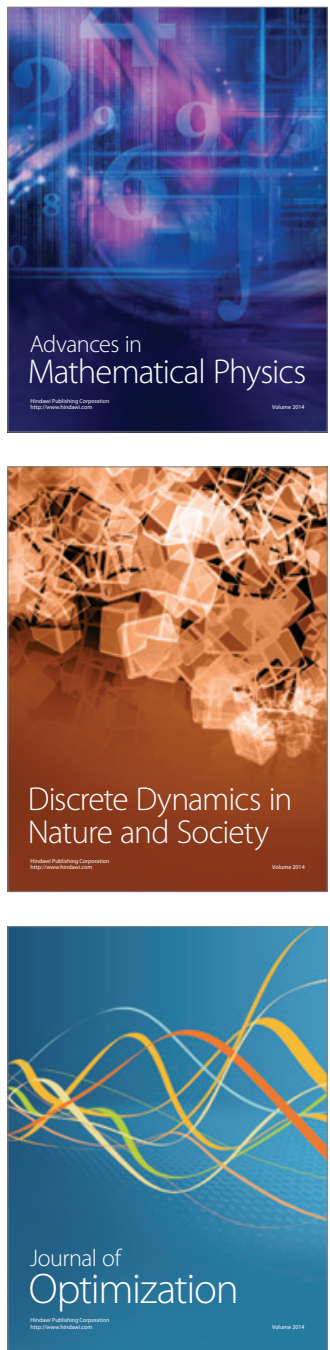